\title{
Cement augmentation of metastatic lesions in the proximal femur can improve bone strength
}

Amelie Sas ${ }^{1}$, Dries Van Camp², Bert Lauwers², An Sermon ${ }^{3}$, G. Harry van Lenthe ${ }^{1}$

${ }^{1}$ Biomechanics Section, Department of Mechanical Engineering, KU Leuven, Belgium

${ }^{2}$ Section Manufacturing Processes and Systems, Department of Mechanical Engineering, KU Leuven, Belgium

${ }^{3}$ Department of Traumatology, University Hospitals Gasthuisberg, Leuven, Belgium and Department of Development and Regeneration, KU Leuven, Belgium

\section{Corresponding author:}

Harry van Lenthe

Biomechanics Section, KU Leuven, Celestijnenlaan 300C, 3001 Leuven, Belgium

harry.vanlenthe@kuleuven.be

$T+3216322595$

\section{Author contributions:}

Amelie Sas: Conceptualization, Methodology, Software, Validation, Formal Analysis, Investigation, Writing - Original draft, Visualization, Project Administration, Funding Acquisition.

Dries Van Camp: Conceptualization, Methodology, Software, Investigation, Writing - Review \& Editing.

Bert Lauwers: Resources, Writing - Review \& Editing.

An Sermon: Conceptualization, Methodology, Investigation, Resources, Writing - Review \& Editing, Supervision, Funding Acquisition

G. Harry van Lenthe: Conceptualization, Methodology, Resources, Writing - Review \& Editing, Supervision, Funding Acquisition.

Declarations of interest: None 


\section{Abstract}

Prophylactic treatment is advised for metastatic bone disease patients with a high risk for fracture. Femoroplasty provides a minimally invasive procedure to stabilize the femur by injecting bone cement into the lesion. However, uncertainty remains whether it provides sufficient mechanical strength to the weight-bearing femur. The goal of this study was to quantify the improvement in bone stiffness, failure load and energy to failure due to cement augmentation of metastatic lesions at varying locations in the proximal femur.

Eight pairs of human cadaveric femurs were mechanically tested until failure in a single-leg stance configuration. In each pair, an identical defect was milled in the left and right femur using a programmable milling machine to simulate an osteolytic lesion. The location of the defects varied amongst the eight pairs. One femur of each pair was augmented with polymethylmethacrylate, while the contralateral femur was left untreated. Digital image correlation was applied to measure strains on the bone surface during mechanical testing.

Only femurs with a critical lesion showed an improvement in failure load and energy to failure due to augmentation. In these femurs, bone strength improved with $28 \%$ ( $\pm 17 \%)$ on average and energy to failure with $58 \%( \pm 41 \%)$, while stiffness did not show a significant improvement. The strain measurements from digital image correlation showed that cement augmentation reinforced the lesion, resulting in reduced strain magnitudes in the bone tissue adjacent to the lesion. The results indicate that femoroplasty may be an effective treatment to prevent fractures in several metastatic bone disease patients. However, the large scatter in the data clarifies the need for developing strategies to identify those patients who will benefit the most from the procedure.

Keywords: Bone metastases; Proximal femur; Fracture; Femoroplasty; Cement augmentation; Digital image correlation 


\section{Introduction}

Bone is one of the most frequent sites of metastasis, particularly in breast and prostate cancer patients. More than $70 \%$ of patients dying of breast cancer showed evidence of metastatic bone disease (MBD) at postmortem examination (Coleman et al., 2006). An important complication of MBD is that it damages and weakens bone, putting the patient at a greater risk for fracture. According to a study by Mirels et al. approximately $30-50 \%$ of bone metastases involving osteolytic lesions lead to pathological fractures (Mirels, 1989). The proximal femur is among the most affected bones (Benca et al., 2017), not only because the incidence of bone metastases is high at this site, but also because it is a weight-bearing bone, suffering from a high risk of fracture (Tian et al., 2016).

To prevent pathological fractures, prophylactic treatment is advised for patients with a high risk for fracture. Several clinical guidelines have been formulated to provide an indication of fracture risk (Van der Linden et al., 2018). Among them, the Mirels' scoring system (Mirels, 1989) seems to be the most commonly used. This scoring system takes size, radiographic appearance and site of the lesion into account, as well as the presence of pain. A score higher than 8 is associated with an impending fracture and indicates the need for prophylactic surgery.

Most current literature supports prophylactic fixation since it is less complex, does much better in terms of quality of life, and results in longer survival compared to the treatment of pathological fractures (Coleman et al., 2006; Derikx et al., 2015; Deschamps et al., 2012). Mechanical stabilization of the bone is typically provided by intra- or extramedullary implants, such as intramedullary nails or plates and screws. Femoroplasty is an alternative, less invasive procedure where bone cement, typically polymethylmethacrylate (PMMA), is injected into the defect created by the tumor. Several clinical studies on femoroplasty as a treatment for bone metastases have already proven the effectiveness of the method to offer immediate pain relief and improved functionality (Anselmetti, 2010; Cazzato et al., 2015; Deschamps et al., 2012; Feng et al., 2016; Plancarte-Sanchez et al., 2013; Tian et al., 2016). However, uncertainty remains whether it provides sufficient mechanical strengthening of the weight-bearing femur (Deschamps et al., 2012) as several reports still indicate a 
substantial risk of fracture even after cementoplasty (Anselmetti, 2010; Deschamps et al., 2012; Tian et al., 2016).

Limited data is available on the mechanical improvement that can be gained with femoroplasty. It has recently been the source of multiple in vitro investigations on cadaver femurs in the application of osteoporosis (Varga et al., 2016). The femoroplasty technique used in osteoporotic proximal femurs is very similar, but cement is injected in broad regions of osteoporotic bone, which is likely to have a different effect than injecting cement locally into an osteolytic metastasis (Kaneko et al., 2007). The improvement in bone strength resulting from filling artificial metastatic lesions with bone cement has been investigated in a limited number of in vitro studies (Kaneko et al., 2007; Palumbo et al., 2014). In these studies the defects have been created by hand with a burr and/or curette, inducing an unavoidable variation in the defect size and shape. Furthermore, both studies mention as a limitation of their work the lack of a control group with an untreated defect to evaluate the direct impact of cementation. To include this comparison, a more repeatable technique for creating the artificial defect is needed. Moreover, only a limited variety of lesion characteristics, such as size and location, have been included in these studies.

Therefore, the aim of this study was to conduct in vitro experiments on human cadaver femurs with artificial lytic lesions at varying locations in the proximal femur and to quantify the mechanical improvement after augmenting these lesions with bone cement. Specifically, we defined the following goals: (1) develop a controlled method for creating the artificial defects to enable direct comparison to a control group with a non-augmented defect, (2) quantify the mechanical effect of cement augmentation on bone stiffness, failure load, energy to failure and local strain magnitudes, (3) measure the cement volume and interdigitation into the surrounding trabecular bone to explore correlations with the mechanical results. 


\section{Methods}

Eight pairs of fresh-frozen $\left(-20^{\circ} \mathrm{C}\right)$ human cadaveric femurs were obtained from the Department of Anatomy (age 60-87 years, 3 males and 5 females, Table 1). Ethical approval was granted by the Ethics Committee of the University Hospitals Leuven (reference number NH019 2018-09-02). Only adult specimens without a fracture or an implant in the proximal femur were included. The femurs were cut at a length of $25 \mathrm{~cm}$ as measured from the top of the head and stripped of soft tissues.

Computed tomography (CT) scans were obtained (Siemens Somatom Force, Siemens AG, Germany) with the following settings: 120 ref kV, 250 ref mAs, slice thickness $0.4 \mathrm{~mm}$, slice increment $0.2 \mathrm{~mm}$, pitch 0.85 and bone kernel. The in-plane pixel size was $0.4 \mathrm{~mm}$. For registration purposes, four tantalum markers $(0.8 \mathrm{~mm})$ were inserted in each femur before scanning at the fovea of the femur head, the top of the greater trochanter, the lesser trochanter and the posterior endosteal surface at the distal end of the femur shaft respectively. During the scan each femur was fixed horizontally in a water basin and placed on top of a calibration phantom (Model 3 CT phantom, Mindways Software Inc., Austin, TX, USA).

\subsection{Artificial cavity creation}

In each femur pair, an identical defect was milled in the left and right femur to simulate a metastatic Iytic lesion. The defects were milled with a programmable five-axis milling machine (Sauer Ultrasonic 70-5, DMG Mori Sauer GmbH, Stipshausen, Germany) to ensure reproducibility of the lesion shape. Two shapes of lesions were defined and programmed: a roughly spherical defect (diameter $15 \mathrm{~mm}$ ) for the femur neck and an ellipsoidal defect (length longest axis $30 \mathrm{~mm}$, other two axes $15 \mathrm{~mm}$ ) for the intertrochanteric region (Fig. 1). Both lesion types were designed to have a cortical opening of 15 $\mathrm{mm}$ diameter to include substantial cortical involvement. Consequently, the upper half of the defects had a cylindrical and conical shape, respectively, to connect the spherical and ellipsoidal bottom half to the cortical opening. This design ensured accessibility of the mill on varying locations of the proximal femur. A $4 \mathrm{~mm}$ diameter ball end mill with a long shaft (103DFORXXL040, Hufschmied 
Zerspanungssysteme $\mathrm{GmbH}$, Bobingen, Germany) was used at a constant spindle speed of $1000 \mathrm{rpm}$.

The feed rate was adapted to the strength of the material to remove, going from $150-250 \mathrm{~mm} / \mathrm{min}$ for cortical bone up to $350 \mathrm{~mm} / \mathrm{min}$ for trabecular bone. After machining, left over debris and bone marrow were removed from the hole with a cotton bud. The femurs were not thawed before machining and were immediately refrozen afterwards to minimize the time of thawing.
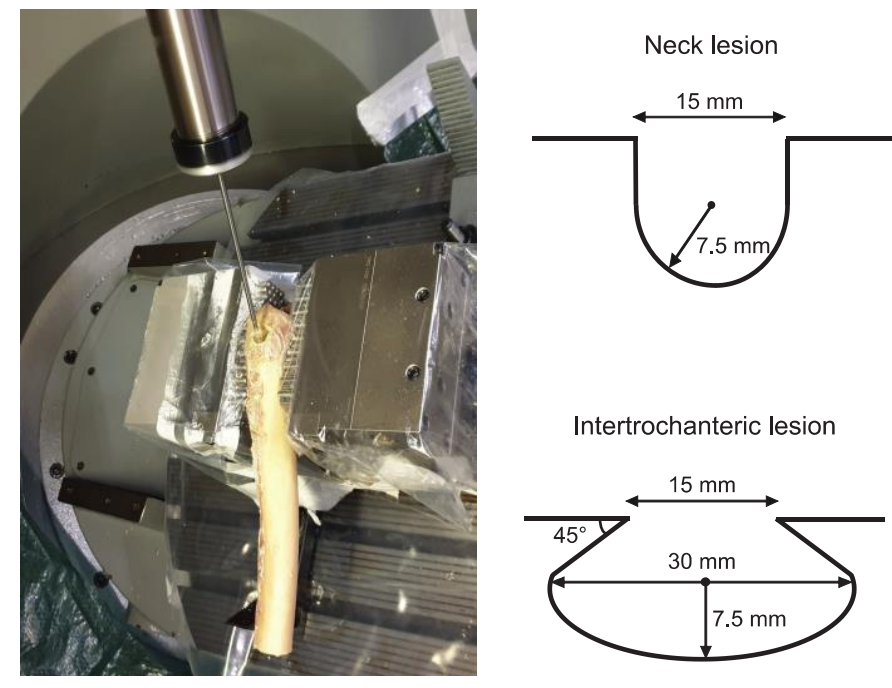

Intertrochanteric lesion

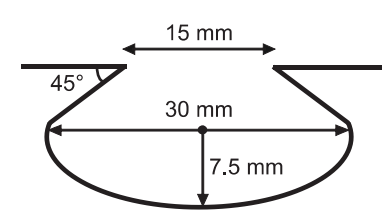

Fig. 1: Experimental setup for milling an intertrochanteric lesion on the lateral aspect of the proximal femur (left). Schematic drawing of the cross-section of the two types of lesions indicating their shape and dimensions (right).

The location of the defects varied amongst the bones. Four pairs received the spherical lesion either in the medial, superior, anterior or posterior aspect of the neck. The other four pairs received the ellipsoidal lesion either in the medial, lateral, anterior or posterior aspect of the intertrochanteric region (Fig. 2). Before milling, the locations of the lesions were manually indicated on each femur pair according to the planned position. Care was taken to achieve an identical position on the left and right femur of each pair. The reference coordinate system of the milling machine was adjusted such that its origin was located at the center of the lesion with the milling axis orthogonal to the surface.

The location and size of the lesions were discussed with a trauma surgeon. All lesions had a "moderate" to "high" risk for a pathological fracture according to the Mirels' scoring system (Mirels, 1989). Since this was a cadaveric study, the contributing factor of pain had to be left out from the scoring metric. Without accounting for this factor, all the lesions reached a score of 7 or 8 . 

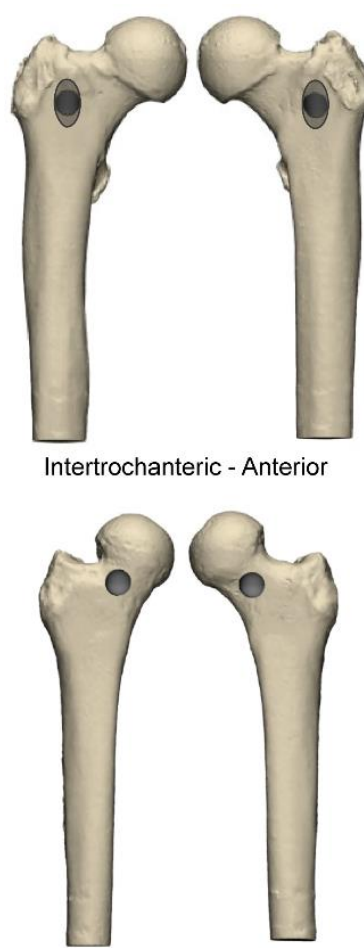

Neck - Anterior
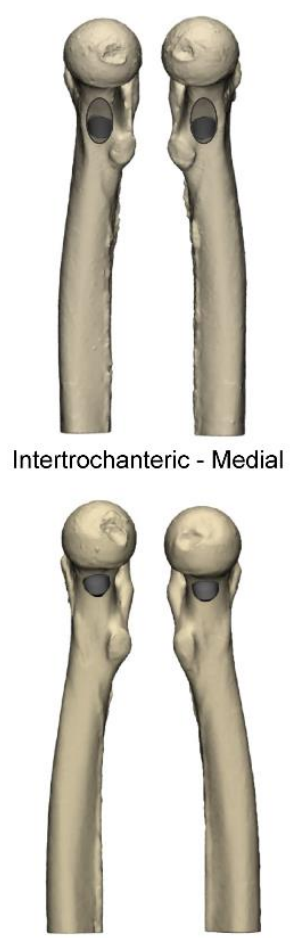

Neck - Medial

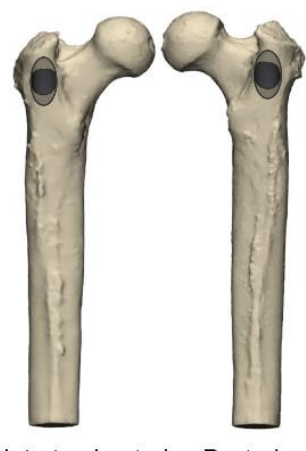

Intertrochanteric - Posterior

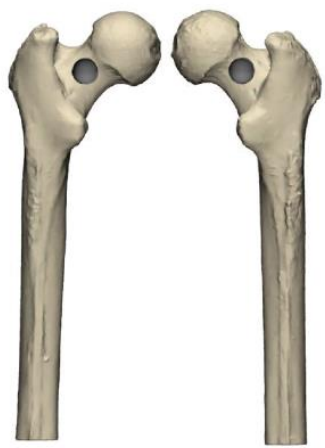

Neck - Posterior

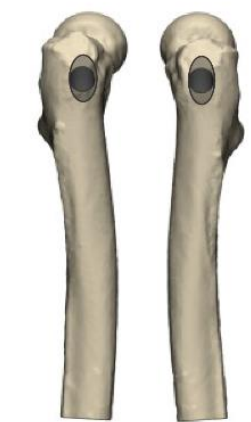

Intertrochanteric - Lateral

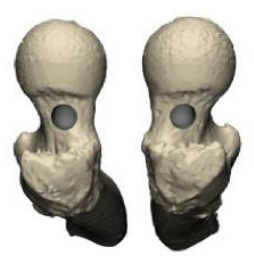

Neck - Superior

Fig. 2: Overview of the locations of the artificial metastatic lesions on the eight pairs of femurs.

\subsection{Cement augmentation}

One randomly selected femur from each pair was assigned for cement augmentation, while the contralateral femur served as a control. Before augmentation, all specimens were thawed at room temperature overnight and CT scanned using the same protocol as described above. PMMA bone cement (Traumacem V+, Synthes $\mathrm{GmbH}$, Oberdorf, Switzerland) was prepared according to the manufacturer's recommendation. Cement was injected directly through the cortical opening. During injection, the cement was distributed and pushed towards the ends of the defect with a curette. Once the hole was completely filled, pressure was manually applied to the cement for approximately 5 min. Another CT scan was made after augmentation to evaluate the cement volume and interdigitation into the surrounding trabecular bone. Based on the position of the tantalum markers in the CT scans, the pre-augmentation scan was aligned to the post-augmentation scan using a point-based registration method. The contour of the lesion was segmented from the pre-augmentation scan and subsequently superimposed on the post-augmentation scan (Fig. 3). To evaluate the thickness of cement interdigitation in the surrounding trabecular bone, the contour of the lesion was compared 
against the contour of the cement, which was segmented from the post-augmentation scan with a simple threshold and hole-filling operation. Uniformly distributed sample points were defined on the lesion contour and for each sample point the distance to the first intersection point with the cement contour was calculated along a direction perpendicular to the lesion surface. The distance measures were averaged over all sample points to provide a single measure of cement interdigitation.
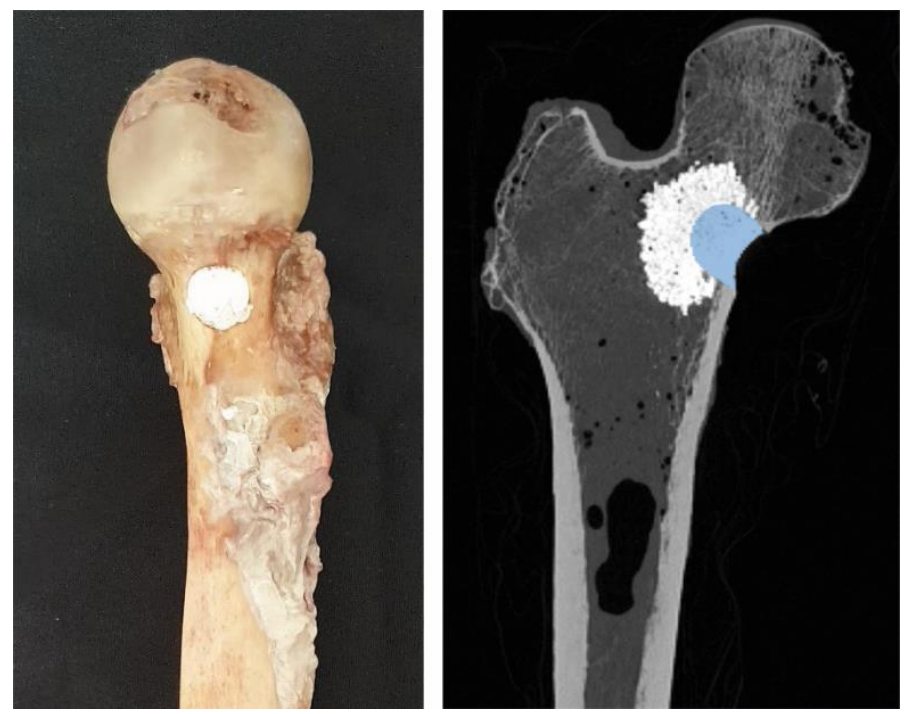

Fig. 3: Picture of a cadaver femur with an augmented lesion in the medial neck (left) and the corresponding CT scan superimposed with a blue mask indicating the extent of the lesion (right).

\subsection{Mechanical testing}

To evaluate the effectiveness of cement augmentation, both femurs of each pair were mechanically tested until failure in a single leg stance configuration. In preparation for mechanical testing, the distal end of each femur was embedded in a PMMA block (Technovit 3040, Heraeus Kulzer, Germany; 5x5x5 $\mathrm{cm}$ cube). The femur was oriented with respect to the embedding block such that the shaft was visually positioned vertically (orthogonal to the bottom plane) and the neck axis parallel to the front plane. The PMMA block was fixed to the mechanical testing machine (Instron 3360, Norwood, MA, USA) in a custom-designed aluminum holder. The holder placed the femur shaft under an axis of $12^{\circ}$ with respect to the loading axis (Kukla et al., 2002) (Fig. 4). To distribute the applied load at the femur head, a custom PMMA cup (50 mm diameter) was molded (not bonded) to each femoral head and placed between the loading plate and the head. The cup was greased to minimize friction and reduce transverse forces/moments. A load was applied to the cup starting with a preload of $50 \mathrm{~N}$, followed 
by 20 sinusoidal preconditioning cycles $(50-500 \mathrm{~N}, 1 \mathrm{~Hz})$ which removed non-linear effects in the initial loading phase. Finally, a ramp load until failure at a rate of $10 \mathrm{~N} /$ second was applied (Derikx et al., 2012). Actuator displacement and load were recorded at $5 \mathrm{~Hz}$.

Bone stiffness, failure force and energy to failure were extracted from the force-displacement curves. Bone stiffness was defined as the steepest slope for a $20 \%$ portion of the force-displacement curve (Benca et al., 2017). Failure force was defined as the maximum force applied and energy to failure as the area under the curve up to the maximal force. The percentage change in stiffness, failure force and energy to failure due to augmentation were quantified for each femur pair and paired t-tests were carried out to identify significant differences between the defect and augmented group. The analysis was evaluated on the full dataset and on a dataset excluding the femur pairs for which the defect femur did not fracture through the created lesion. The rationale for excluding the latter cases was that in these cases the created lesions did not represent the critical weakest location of the femur. Finally, linear regression analyses were performed between the mechanical data, cement volume and cement interdigitation. All statistical analyses were performed in MATLAB R2017a (MathWorks, Natick, MA) and $p$-values $<0.05$ were considered statistically significant.
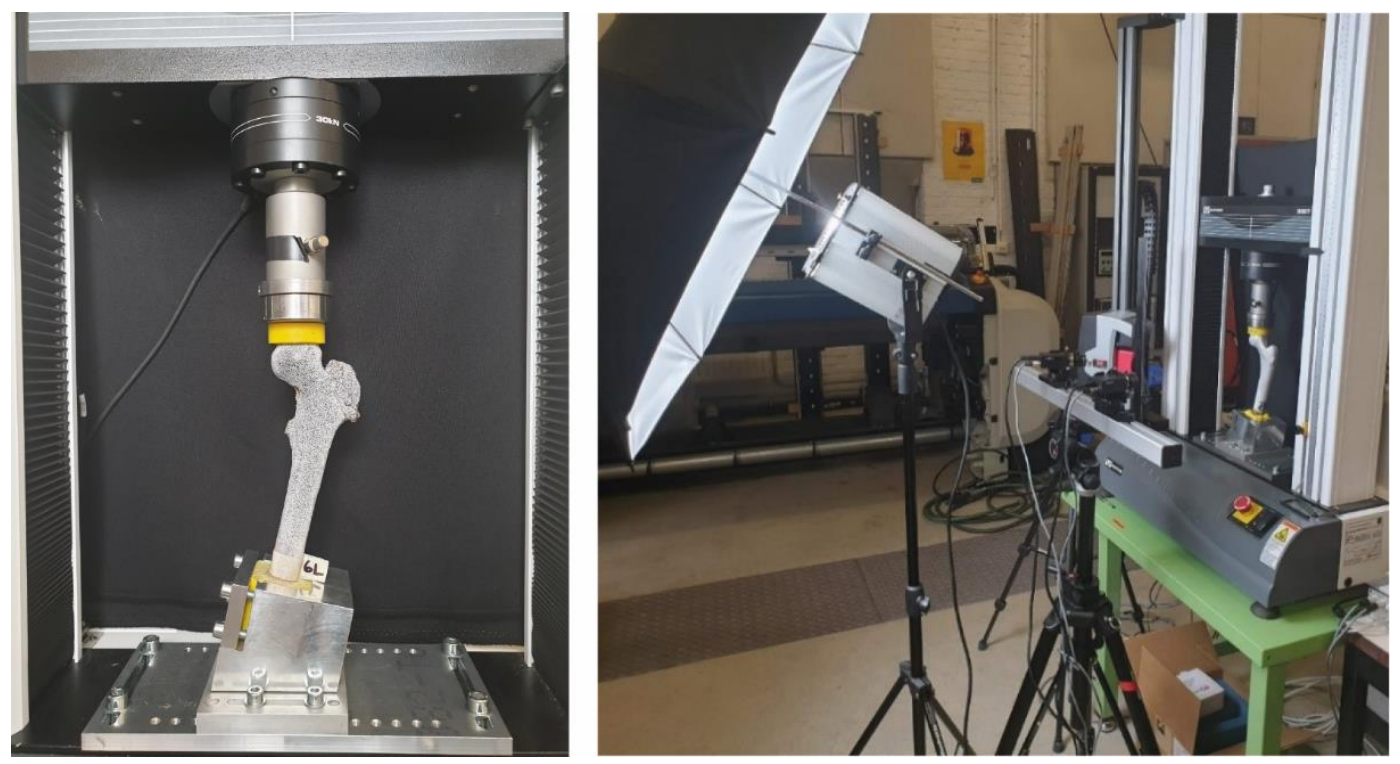

Fig. 4: Images of the experimental setup. The distal part of the femur is fixed onto the test machine with a holder that positions the femur such that its shaft is under an angle of $12^{\circ}$ with respect to the loading axis (left). Two cameras and a cold light source are positioned in front of the test device for DIC recording (right). 


\subsection{Digital image correlation}

During the mechanical test, two cameras recorded the anterior surface of the femur for digital image correlation (DIC) measurements of bone surface strains. DIC is a three-dimensional, non-contact optical technique that allows to measure full-field contour, displacement and strain over the surface of an object and has relatively recently been introduced for strain measurements during in vitro mechanical testing of cadaver femurs (Gilchrist et al., 2013; Grassi et al., 2014). Prior to testing, the anterior surface of the femur was thoroughly cleaned from soft tissues using scalpels and fine sandpaper. A matt white background was applied using solvent-based spray paint to increase the contrast. On top of the white layer, a black random speckle pattern was applied with an airbrush. We aimed for a 6 by 6 px speckle size (Grassi et al., 2014), resulting in a physical speckle size of approximately $0.4 \mathrm{~mm}$ for the adopted camera setup.

The DIC set-up (Isi-sys GmbH, Kassel, Germany) (Fig. 4) consisted of two cameras (Grasshopper3, Flir Systems Inc., $5 \mathrm{Mpx}$ ) to record the test at 5 frames per second. The two cameras were placed in front of the anterior aspect of the tested specimens, with a baseline camera distance of $220 \mathrm{~mm}$ and a pan angle of approximately $30^{\circ}$. A high-intensity cold light source was used to provide a diffuse light to the specimens, allowing for a smaller exposure time and higher aperture of the cameras. Before starting to load the specimens until fracture, images were recorded for $5 \mathrm{~s}$ at a constant force equal to the preload to serve for noise analysis.

Vic-3D software (Correlated Solutions Inc., Irmo, SC) was used to perform DIC on the acquired images to retrieve the displacement and strain at the anterior surface of the femur. The selected frames of interest under loading were at a force of $50 \%$ and $75 \%$ of the maximal force. Additionally, a prefracture frame was selected as the frame prior to the frame where a crack could be detected by naked eye. To quantify the noise, 15 frames were selected in the middle section of the $5 \mathrm{~s}$ recording in undeformed state. Correlation was performed using a subset size of $29 \mathrm{px}$ and a step size of $6 \mathrm{px}$. All the frames were compared to the same reference picture, the frame immediately before the selected images of noise recording. Before correlation, the images were filtered using a low-pass filter 
(binomial, [ 0.250 .50 .25$]$ ) in the $\mathrm{X}$ and $\mathrm{Y}$ direction to remove high frequency information and reduce potential aliasing effects. Engineering strain was then calculated from the displacement vectors using a decay filter of $5 \mathrm{px}$. The major and minor principal strains were visualized for the selected frames of interest.

The strain magnitudes were compared between the defect and augmented femur of each pair at identical loads equal to $50 \%$ and $75 \%$ of the maximal force of the defect femur. In the four femur pairs where the lesion was visible on the DIC images (neck anterior and medial, intertrochanter anterior and medial), the strain magnitudes were also compared in a region adjacent to the lesion, which was manually outlined on the images. Specifically, a circular region of interest was drawn, centered at the cortical opening of the lesion and with a diameter equal to two times the diameter of the cortical opening. For the medial neck and intertrochanteric lesion, a semicircle was drawn since they were only visible from the side (Fig. 9). As an indicator of the noise level, the mean and standard deviation of the major and minor principal strain were calculated over all datapoints of the 15 noise frames.

\section{Results}

\subsection{Cement volume and interdigitation}

The injected cement volume ranged from 5.4 to $9.5 \mathrm{ml}$ (Table 1). Cement interdigitated into the surrounding trabecular bone over a mean distance ranging from 1.35 to $6.02 \mathrm{~mm}$, with an average value of $3.82 \mathrm{~mm}$ (Table 1). Less interdigitation was observed in the intertrochanteric lesions (mean distance $2.59 \mathrm{~mm}$ ) compared to the neck lesions (mean distance $5.05 \mathrm{~mm}$ ).

Table 1: Subject data and cement distribution parameters

\begin{tabular}{ll|lllllll}
\hline Lesion type & Sex & $\begin{array}{l}\text { Age } \\
\text { (years) }\end{array}$ & $\begin{array}{l}\text { Weight } \\
\text { (kg) }\end{array}$ & $\begin{array}{l}\text { Augmented } \\
\text { femur }\end{array}$ & $\begin{array}{l}\text { Cement } \\
\text { volume (m) })\end{array}$ & $\begin{array}{l}\text { Cement } \\
\text { interdigitation } \\
\text { (mean } \pm \text { SD; mm) }\end{array}$ \\
\hline Neck & Medial & F & 82 & 110 & Right & 7.0 & 6.02 & \pm 3.16 \\
& Anterior & F & 60 & 54 & Right & 7.5 & 5.26 & \pm 1.97 \\
& Superior & M & 87 & 70 & Left & 7.9 & 5.11 & \pm 3.49 \\
& Posterior & F & 84 & 69 & Left & 7.7 & 3.80 & \pm 3.61 \\
Inter- & Medial & M & 70 & 45 & Right & 9.5 & 3.15 & \pm 3.55 \\
troch & Anterior & M & 62 & 110 & Right & 5.8 & 2.17 & \pm 1.93 \\
& Lateral & F & 63 & 63 & Left & 5.4 & 1.35 & \pm \\
& Posterior & F & 68 & 90 & Left & 9.4 & 3.70 & \pm 2.44 \\
\hline
\end{tabular}




\subsection{Mechanical data}

Five out of eight defect femurs fractured through the created lesion. In the corresponding contralateral, augmented femurs, fractures were located at the bone-cement interface. The three femurs that did not fracture through the lesion had an intertrochanteric lesion on the anterior, posterior and lateral side respectively. In the latter, an intertrochanteric neck fracture was observed with a fracture line located more medially than the lesion. A similar fracture pattern was observed in their corresponding contralateral, augmented femur.

A sudden crack formation was observed for all femur pairs. Only one femur pair with the anterior neck lesion showed a different failure behavior, i.e. a compression fracture where the bone slowly collapsed under pressure without obvious crack formation. Videos and images of the fractures can be found in the supplementary material.

Cement augmentation increased the stiffness, failure force and energy to failure in almost all femur pairs (Table 2, Fig. 5). The increase was more pronounced in the restricted dataset including only the five femur pairs that fractured through the lesion (Table 2, Fig. 6). Stiffness increased non-significantly by $7 \%($ SD $10 \%, p=0.2$ ) on average in the full dataset and by $8 \%$ (SD $8 \%, p=0.1$ ) in the restricted dataset. Failure force increased significantly by $19 \%$ (SD 19\%, $p<0.05$ ) and $28 \%$ (SD 17\%, $p<0.01$ ) on average in the full and restricted dataset respectively. Analogously, a significant increase in energy to failure was achieved by $39 \%$ (SD 41\%, $p<0.01$ ) and $58 \%$ (SD 41\%, $p<0.01$ ) respectively.

Correlation analysis revealed a relation between the effect of augmentation and the mechanical properties of the non-augmented defect femur (Fig. 7). Matched pairs with a weaker defect femur showed a larger relative increase in failure load and energy to failure. No significant correlations were found between the mechanical data and cement parameters, i.e. cement volume and interdigitation distance. 

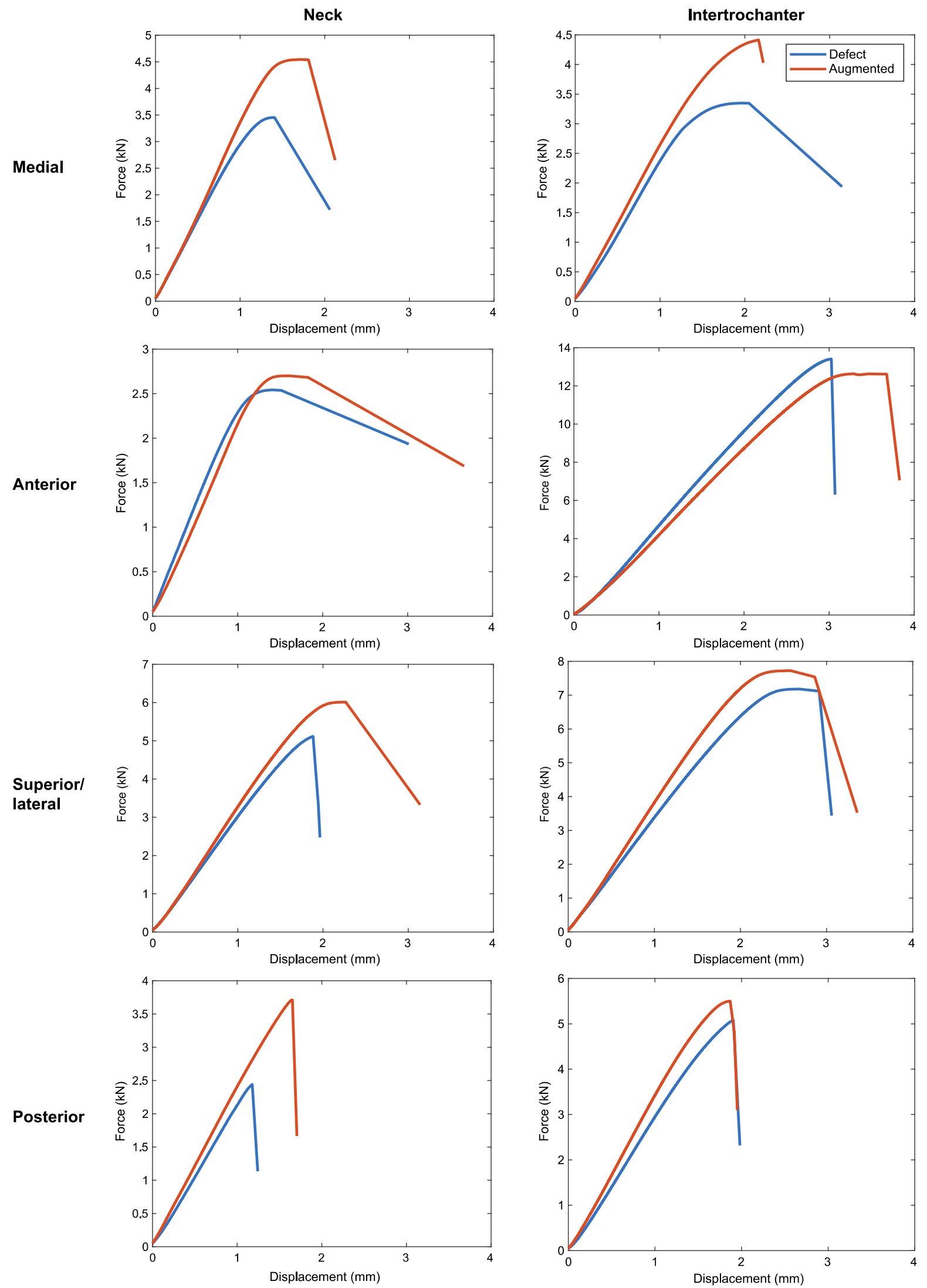

Figure 5: Force-displacement curves of the eight femur pairs included in this study. A higher maximal force was observed for the augmented femur compared to the defect femur, except for the specimen with an anterior-intertrochanteric lesion. No significant differences were observed for the stiffness (slope). 
Table 2: Results from mechanical testing

\begin{tabular}{|c|c|c|c|c|c|c|c|c|c|c|c|}
\hline \multicolumn{2}{|c|}{ Lesion type } & \multirow{2}{*}{$\begin{array}{l}\text { Lesion } \\
\text { failure }\end{array}$} & \multicolumn{3}{|c|}{ Stiffness (N/mm) } & \multicolumn{3}{|c|}{ Failure force (kN) } & \multicolumn{3}{|c|}{ Energy to failure $(\mathrm{J})$} \\
\hline & & & Def & Aug & $\Delta(\%)$ & Def & Aug & $\Delta(\%)$ & Def & Aug & $\Delta(\%)$ \\
\hline \multirow[t]{4}{*}{ Neck } & Medial & Yes & 3.07 & 3.58 & 17 & 3.45 & 4.54 & 32 & 2.80 & 4.57 & 63 \\
\hline & Anterior & Yes & 2.41 & 2.27 & -6 & 2.54 & 2.70 & 6 & 2.19 & 2.54 & 16 \\
\hline & Superior & Yes & 3.10 & 3.46 & 12 & 5.11 & 6.01 & 18 & 5.11 & 7.53 & 47 \\
\hline & Posterior & Yes & 2.20 & 2.41 & 9 & 2.44 & 3.71 & 52 & 1.41 & 3.17 & 125 \\
\hline \multirow{4}{*}{$\begin{array}{l}\text { Inter- } \\
\text { troch }\end{array}$} & Medial & Yes & 2.53 & 2.69 & 6 & 3.35 & 4.41 & 32 & 4.00 & 5.59 & 40 \\
\hline & Anterior & No & 5.20 & 4.68 & -10 & 13.41 & 12.63 & -6 & 21.33 & 22.69 & 6 \\
\hline & Lateral & No & 3.43 & 3.97 & 16 & 7.18 & 7.72 & 8 & 11.30 & 11.35 & 0 \\
\hline & Posterior & No & 3.12 & 3.56 & 14 & 5.08 & 5.50 & 8 & 5.08 & 5.64 & 11 \\
\hline
\end{tabular}

Def $=$ Defect; Aug = Augmented; $\Delta=$ percentage change

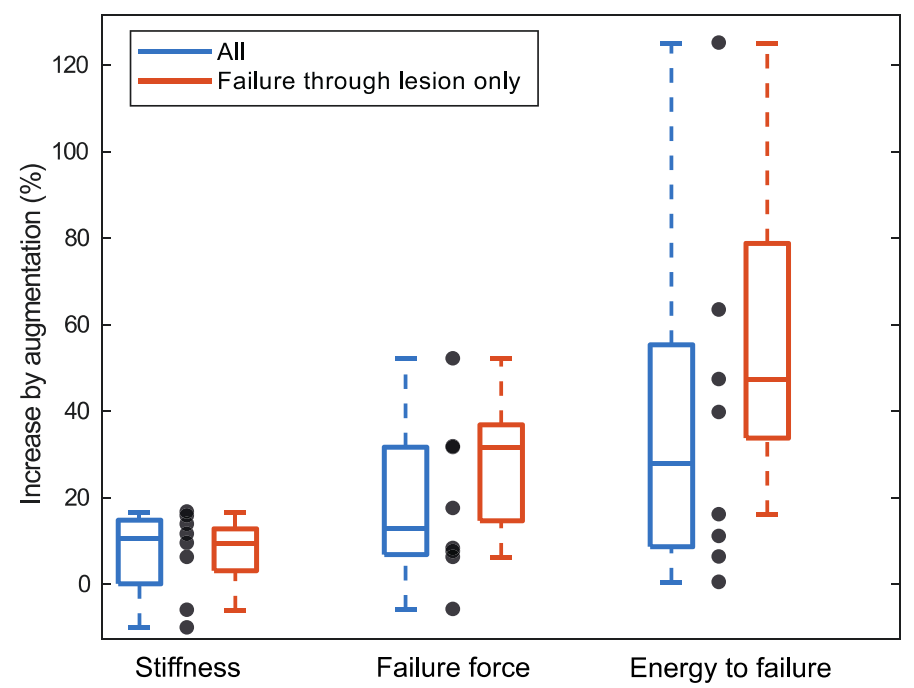

Fig. 6: Boxplots of the percentage increase in stiffness, failure force and energy to failure due to cement augmentation. The increase in failure force and energy to failure was more pronounced in the dataset including only the femurs that fractured through the lesion compared to the full dataset.

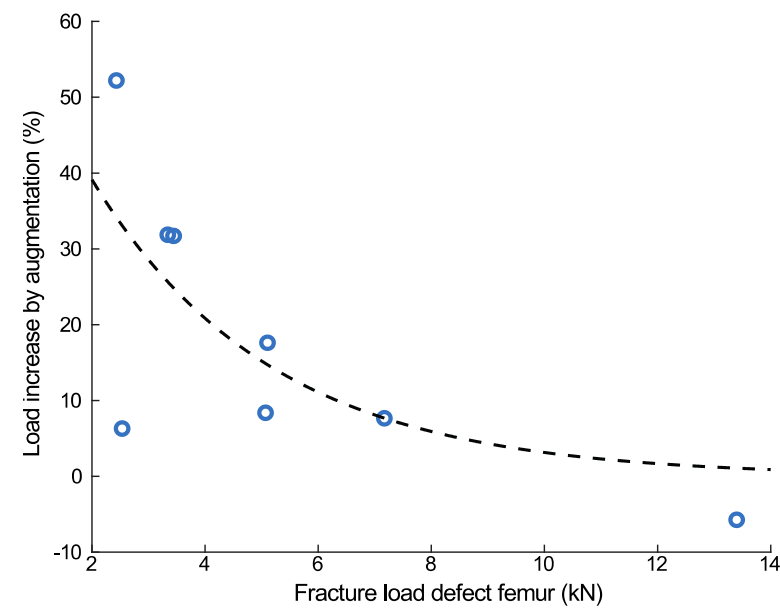

A

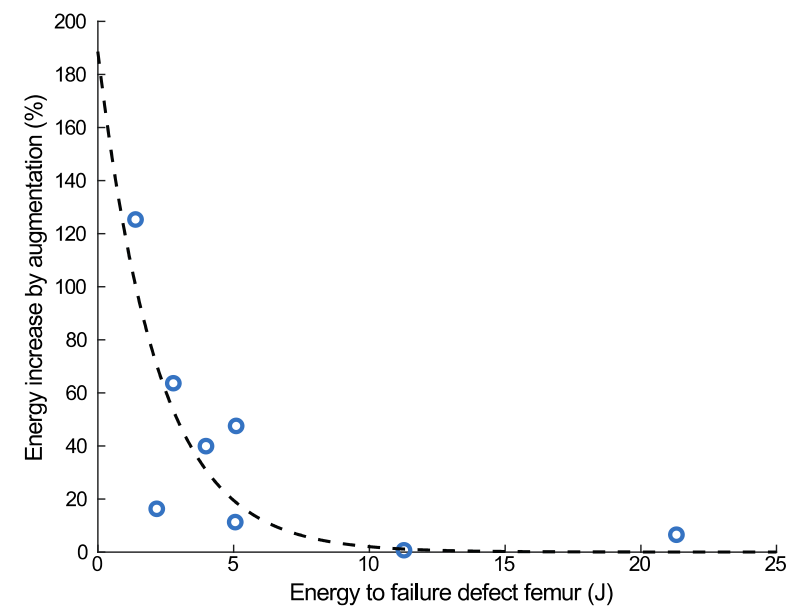

B

Fig. 7: The percentage increase in failure load $(A)$ and energy to failure (B) due to augmentation were related to the mechanical properties of the non-augmented, defect femur. Femur pairs with a weaker defect femur showed a larger relative increase. 


\subsection{Digital image correlation}

The major and minor principal strain magnitudes consistently increased in absolute value with increasing applied load (Fig. 8). The images at $50 \%$ and $75 \%$ of the maximal force of the defect femur showed a physiological strain distribution with tensile strains mainly building up in the femoral neck region and compressive strains in the cortex on the medial side. In the pre-fracture image, the onset of crack formation was observed. At several points corresponding to the starting location of the crack, the strain magnitudes exceeded the bone yield limit of $7300 \mu \varepsilon$ in tension or $10400 \mu \varepsilon$ in compression (Bayraktar et al., 2004). However, in some cases (7/16) the camera recording frequency was too slow to visualize the initiation of the crack.

No clear differences were observed between the defect and augmented femur for the average strain magnitudes in the total region of interest. However, in the region adjacent to the lesion the major and minor principal strain magnitudes were consistently higher for the untreated lesion compared to the augmented lesion (Table 3). The differences were most pronounced for the anterior neck lesion and the medial intertrochanteric lesion (Figure 9).

DIC noise was calculated for each femur from 15 images under preload before the start of the ramp load to failure. The average major principal strain ranged from $119 \pm 163 \mu \varepsilon$ to $177 \pm 174 \mu \varepsilon$. The average minor principal strain ranged from $-165 \pm 174 \mu \varepsilon$ to $-114 \pm 151 \mu \varepsilon$.

Table 3: Major $\left(e_{1}\right)$ and minor $\left(e_{2}\right)$ principal strain magnitudes in the region adjacent to the lesion

\begin{tabular}{|c|c|c|c|c|c|c|c|c|}
\hline \multirow{2}{*}{ DIC frame + Lesion type } & \multicolumn{4}{|c|}{$e_{1}$ (mean \pm SD; $\left.\mu \varepsilon\right)$} & \multicolumn{4}{|c|}{$e_{2}($ mean $\pm S D ; \mu \varepsilon)$} \\
\hline & \multicolumn{2}{|r|}{ Def } & \multicolumn{2}{|c|}{ Aug } & \multicolumn{2}{|c|}{ Def } & \multicolumn{2}{|c|}{ Aug } \\
\hline \multicolumn{9}{|l|}{$50 \% F_{\max , \text { defect }}$} \\
\hline Neck - Anterior & 2425 & \pm 5681 & 1212 & \pm 890 & 2297 & \pm 1862 & 1357 & \pm 885 \\
\hline Neck - Medial & 360 & \pm 906 & 250 & \pm 654 & 2576 & \pm 735 & 2059 & \pm 683 \\
\hline Intertroch - Anterior & 396 & \pm 538 & 281 & \pm 429 & 1341 & \pm 676 & 1212 & \pm 532 \\
\hline Intertroch - Medial & 955 & \pm 1955 & 115 & \pm 585 & 2404 & \pm 2103 & 2022 & \pm 745 \\
\hline \multicolumn{9}{|l|}{$75 \% F_{\max , \text { defect }}$} \\
\hline Neck -Anterior & 4780 & \pm 11586 & 2153 & \pm 1723 & 3870 & \pm 3574 & 2279 & \pm 1490 \\
\hline Neck - Medial & 599 & \pm 1610 & 406 & \pm 1052 & 4192 & \pm 1456 & 3151 & \pm 1034 \\
\hline Intertroch - Anterior & 539 & \pm 777 & 446 & \pm 626 & 2116 & \pm 1027 & 1883 & \pm 814 \\
\hline Intertroch - Medial & 1622 & \pm 3310 & 233 & \pm 888 & 4219 & \pm 3709 & 3318 & \pm 1188 \\
\hline
\end{tabular}

Def $=$ Defect; Aug $=$ Augmented 

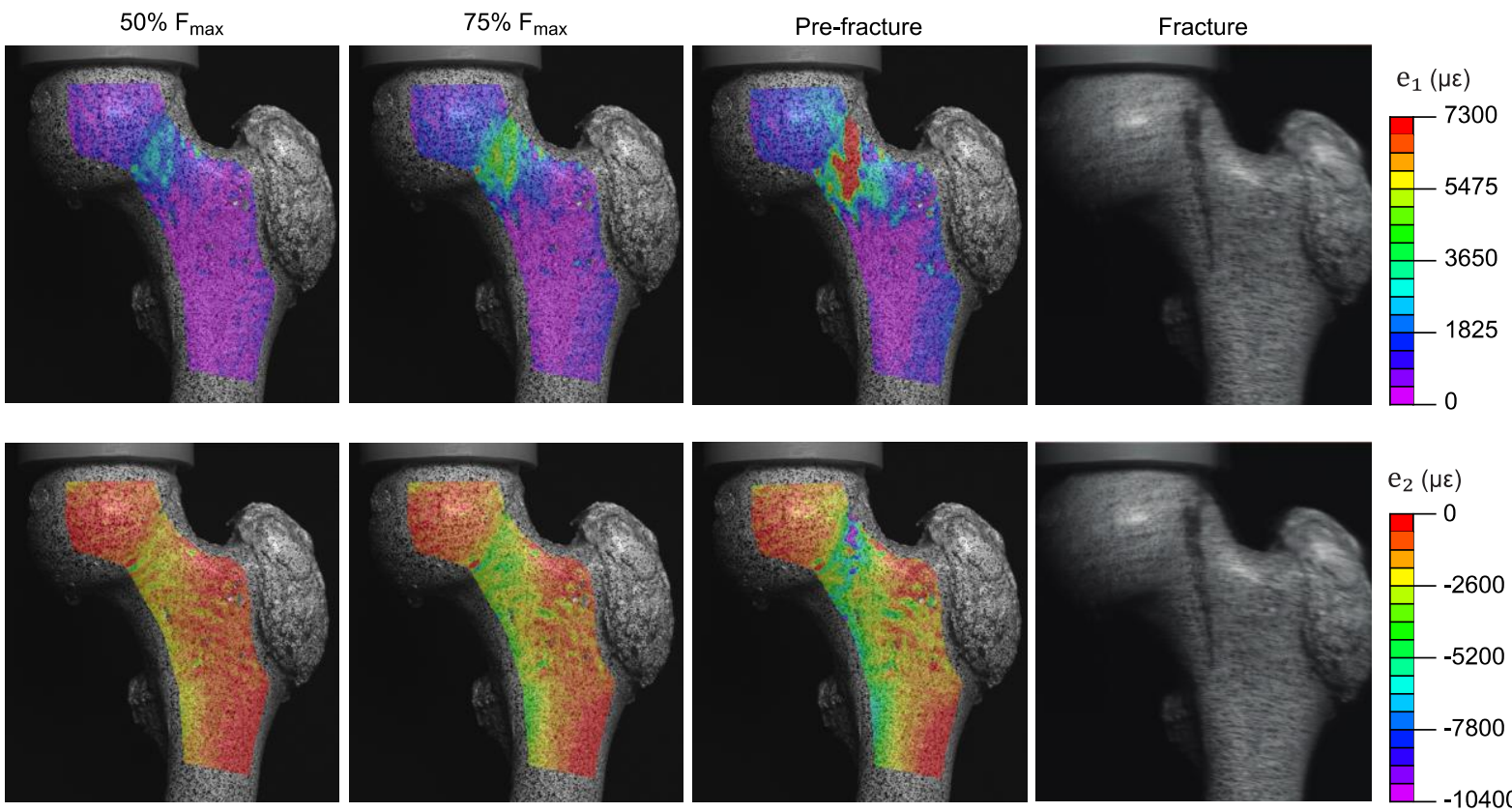

Fig. 8: The major and minor principal strains measured by DIC are superimposed on the raw camera pictures for the augmented femur with a posterior neck lesion. The strain magnitudes are visualized at three time points: at $50 \%$ and $75 \%$ of the maximal force, and at the frame immediately before the fracture frame where a crack was detected by naked eye.
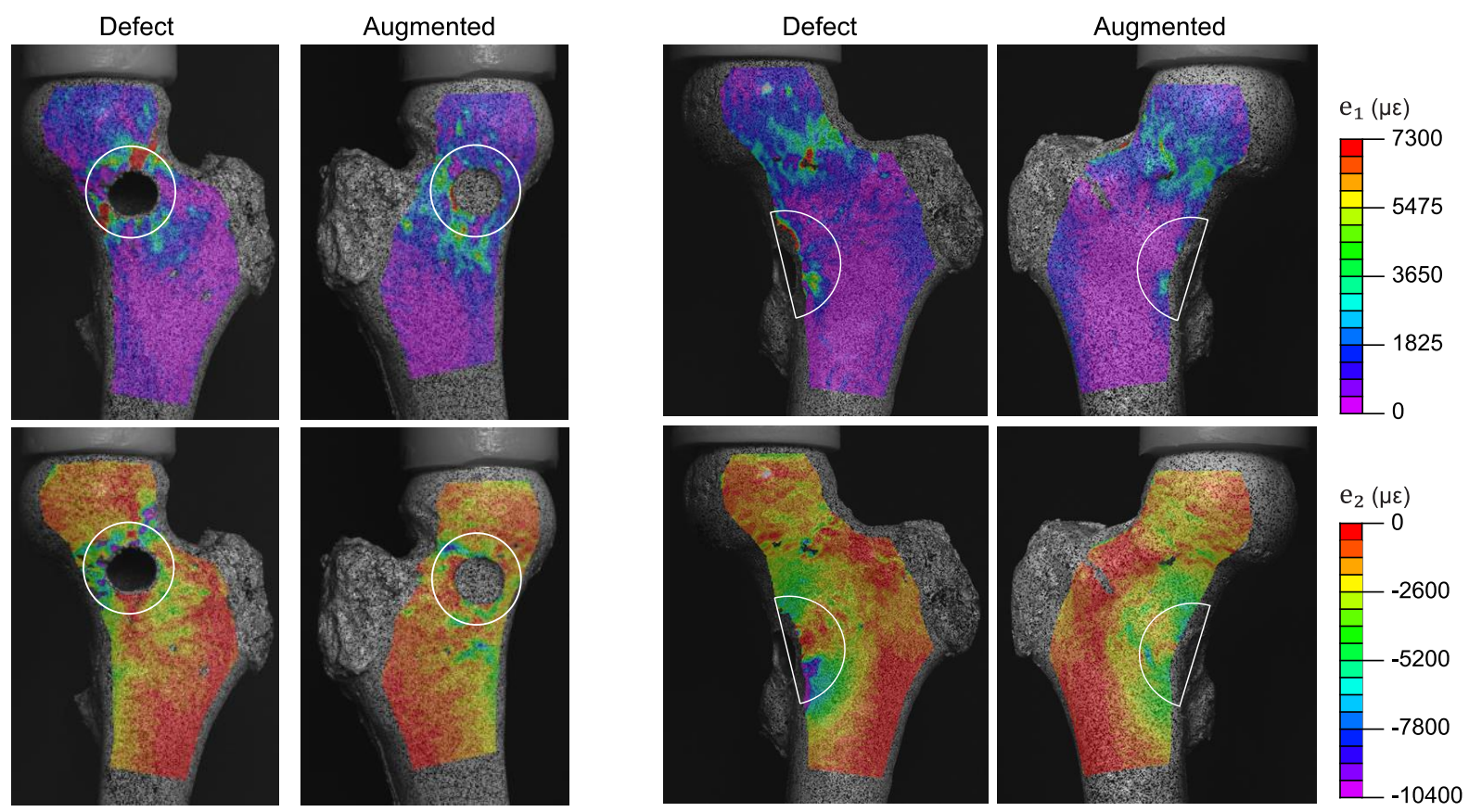

A

B

Fig. 9: The major and minor principal strains measured by DIC are superimposed on the raw camera pictures for the femur pair with an anterior neck lesion (A) and a medial intertrochanteric lesion (B). The strain magnitudes for the defect and augmented femur in each pair are visualized at the same force equal to $75 \%$ of the maximal force of the defect femur. Reduced strain magnitudes are observed for the augmented femur in the region adjacent to the lesion (white contour). 


\section{Discussion}

Through mechanical experiments on human cadaver femurs, we demonstrated that augmentation with PMMA improves the strength and energy to failure of proximal femurs with lytic, metastatic lesions. Strain measurements from digital image correlation showed that cement augmentation reinforced the lesion, resulting in reduced strain magnitudes in the neighborhood of the lesion.

As opposed to conventional surgical fixation, femoroplasty could provide important benefits for the patient, such as a shorter and less invasive procedure, less pain and discomfort, and reduced hospital stay and recovery time (Deschamps et al., 2012). The interest for such minimally invasive, prophylactic procedures for patients with bone metastases has increased in recent years as the number of metastatic patients with long-term survival has increased due to the improved cancer treatment methods (Ratasvuori et al., 2013). When patients are living longer with the disease, it gives them more time to develop secondary complications such as pathological fractures.

The effect of cement augmentation on strength and energy to failure was more pronounced for critical lesions that jeopardized the mechanical integrity and caused the femur to fracture through the lesion. Three defect femurs did not fracture through the created lesion, even though they had Mirels' scores similar to that of the other femurs ( 7 or 8 without accounting for the pain factor). Apparently, the lesions in these femurs did not act as a critical weak spot. In line with these findings, almost no improvements in stiffness, failure load or energy to failure due to cement augmentation were observed for these three cases.

The minimal improvement after augmentation in these three femurs was also in accordance to the observed correlation between the augmentation effect and initial strength properties (Fig. 6). Stronger femurs experienced less relative strengthening than weaker femurs, typically femurs with a critical lesion. A similar trend was observed previously by Varga et al. in the evaluation of prophylactic augmentation for osteoporotic femurs (Varga et al., 2016). Analogously to this study, we observed a decreasing relation similar to $1 / X$, which could also be expected since the initial strength appears in the denominator when measuring the relative effect. 
The femur pair with the anterior neck lesion showed a behavior that deviated from this trend. Although it was one of the weakest femur pairs, only a slight improvement in failure load and energy to failure was found. We hypothesize that this different behavior is related to difference in failure mode; this femur pair slowly collapsed under pressure without obvious crack formation, contrary to all other femurs in the dataset.

Cement interdigitated into the surrounding bone over a distance of almost $4 \mathrm{~mm}$ on average. More interdigitation occurred in neck lesions compared to the intertrochanteric lesions. A potential reason for this is that the neck lesions were almost fully surrounded by trabecular bone, while the intertrochanteric lesions were more surrounded by impenetrable cortical bone, since the cortical layer was much larger at these locations. Cement interdigitation and total volume showed no correlation with the mechanical results.

Using DIC, we were able to visualize physiological strain distributions on the femur surface. The DIC measurements showed that cement augmentation reduced the strain magnitudes in the region adjacent the lesion. These reduced strain levels could have been expected since the goal of cement augmentation is to provide mechanical stabilization to the bone surrounding the lesion. Yet, this comparison was only possible for half of the femur pairs since the lesions in the remaining pairs were located on the backside of the femur and were therefore not visible for DIC. In most cases, crack initiation was visible in the image immediately before fracture, although the camera speed was often too slow to capture it. A higher camera speed is needed if one aims to visualize the crack propagation in detail, but this was out of the scope of this study. The physiological strain results at $50 \%$ and $75 \%$ of the maximal force are useful data that can serve for the validation of numerical models. Indeed, Grassi et al. have previously demonstrated how they validated their FE models against DIC results (Grassi et al., 2016). The full-field strain measurements from DIC provide a more comprehensive validation as opposed to the limited number of measurements typically collected using strain gauges (Grassi et al., 2016). The average DIC noise levels in this study were in line with the noise values measured by Grassi et al. (Grassi et al., 2014), although we found slightly larger standard deviations. 
A potential reason is that we measured noise from the speckle pattern on the femur surface itself, while Grassi et al. measured it from a flat control plate beside the femur which allows a more ideal speckle pattern.

It is difficult to compare our results directly with previous cadaver studies on prophylactic treatment with femoroplasty. First of all, analogous studies on metastatic bone disease, creating artificial lytic lesions and augmenting them with bone cement (Kaneko et al., 2007; Palumbo et al., 2014), did not compare the effect of augmentation against a control group with untreated defects but instead compared it against an intact group and an internally fixated group with compression screws, respectively. Both studies mention the lack of a comparison with a negative control group as a limitation in their study. To the best of our knowledge, we are the first ones to conduct such a comparison, which became possible thanks to the controlled method for creating defects using a programmable mill. Alternatively, when comparing with studies on the augmentation of osteoporotic bone, the methodology differs since the authors do not create defects into the femur. Nevertheless, we can observe some similar trends. Stiffness always showed only minimal improvement, while the largest effects were observed for the failure load and energy to failure (Fliri et al., 2013; Sutter et al., 2010). While for critical lesions we observed an average improvement in failure load and energy to failure of respectively $28 \%$ and $58 \%$, respectively, others have typically reported larger values for the relative improvement, especially in energy to failure (Basafa et al., 2015; Beckmann et al., 2011; Fliri et al., 2013; Heini et al., 2004; Sutter et al., 2010). Amongst others, this can be explained by the differing loading condition of a sideways fall instead of single leg stance. This discrepancy was clearly demonstrated by Heini et al. who found an increase in energy to failure of $48 \%$ in single-leg stance configuration opposed to $188 \%$ in sideways fall for the same augmentation method (Heini et al., 2004). Analogously, fracture load increased with $21 \%$ opposed to $82 \%$ respectively. In our study, a single-leg stance configuration was chosen since MBD patients often suffer from spontaneous fractures, even during normal daily activities such as walking (Eggermont et al., 2018). Finally, the observed fracture 
patterns of the augmented femurs often involved the bone-cement interface (Kaneko et al., 2007; Palumbo et al., 2014), as was also the case in this study.

A first limitation of the study was the use of artificial cavities, which have a simplified shape compared to actual metastatic lesions with an arbitrary shape. However, considering the goal of this study to create identical lesions in the left and right femur of a matched pair, we decided to use programmable defects and therefore had to make a certain assumption on the lesion shape. Another simplification was filling the lesions from the cortical opening instead of injecting cement through a needle or cannula inserted from the lateral cortex. The remaining canal after retraction of the needle might additionally weaken the bone. However, this weakening effect would be reduced by also filling the canal with bone cement, and previous studies have indicated that the effect on bone strength is minimal (Kaneko et al., 2007).

Large variation was observed in the data, which made it hard to quantify the general effectiveness of cement augmentation. Part of this variation was most likely related to the differing lesion sizes and locations in this study. Yet, with the small dataset of only one femur pair per lesion, we were not able to say whether one lesion type benefits more than the other. Large population-based analyses may therefore be needed to assess the general effectiveness and potentially relate it to the type of lesions (Varga et al., 2016). Numerical methods such as finite element analysis (FEA) could serve for this purpose. They open up the way for retrospective analyses of large available CT or MRI databases of patients with real bone metastases (Eggermont et al., 2019; Sternheim et al., 2018). Alternatively, they enable to compare the effect of lesion location by simulating artificial defects at varying locations in the FE model (Rajapakse et al., 2019). Currently, FE models have already shown to be strong predictors of fracture risk in patients with bone metastases (Derikx et al., 2012; Eggermont et al., 2019). By extending these models to simulate femoroplasty, they could become important tools to both assess the need for prophylactic augmentation and identify patients for whom femoroplasty would be appropriate. Extensive validation studies are however required to prove the accuracy and precision of simulating the treatment with FEA. 
A remaining uncertainty is what level of strength improvement is required to prevent actual fractures. A suggested way of quantifying this is by calculating the load-to-strength ratio, where the load can be estimated from the patient's weight and height and the strength can be predicted with FE analysis (Kok et al., 2019; Varga et al., 2016). In theory, cement augmentation would be effective if it would reduce this ratio to a value smaller than 1 . Based on the results from a prospective case study by Orwoll et al. (Orwoll et al., 2009), who found an average load-to-strength ratio of 1.13 for fracture cases, this would mean that on average an increase of $13 \%$ in strength would suffice (Kok et al., 2019). Yet, there was a wide range in the load-to-strength ratios (from 0.2 up to 2.4), indicating that many patients would require a larger strength increase. Hence, this load-to-strength ratio provides a useful concept to quantify the effectiveness of prophylactic treatment in preventing fractures, but it requires further refinement to improve the accuracy (Varga et al., 2016).

In summary, this study has demonstrated that cement augmentation of critical, metastatic lesions in the proximal femur improves bone strength and energy to failure. DIC allowed us to capture a detailed strain map of the entire proximal femoral surface, which showed that cement augmentation reduced the strain magnitudes in the region adjacent the lesion. The results indicate that femoroplasty may be an effective treatment method to prevent fractures in several metastatic bone disease patients; however, strategies should be developed to identify these patients who will benefit the most from the procedure.

\section{Acknowledgements}

This work was funded by the Research Foundation Flanders FWO (application number 1S34218N). We thank Antonio Cutolo for his technical support in mechanical testing and Walter Coudyzer for acquiring the CT scans. Bone cement was generously provided by DePuySynthes.

\section{References}

Anselmetti, G.C., 2010. Musculoskeletal Interventions Osteoplasty Percutaneous Bone Cement 
Injection beyond the Spine. Semin. Intervent. Radiol. 27, 199-208.

Basafa, E., Murphy, R.J., Otake, Y., Kutzer, M.D., Belkoff, S.M., Mears, S.C., Armand, M., 2015.

Subject-specific planning of femoroplasty: An experimental verification study. J. Biomech. 48 , 59-64. https://doi.org/10.1016/j.jbiomech.2014.11.002

Bayraktar, H.H., Morgan, E.F., Niebur, G.L., Morris, G.E., Wong, E.K., Keaveny, T.M., 2004.

Comparison of the elastic and yield properties of human femoral trabecular and cortical bone tissue. J. Biomech. 37, 27-35. https://doi.org/10.1016/S0021-9290(03)00257-4

Beckmann, J., Springorum, R., Vettorazzi, E., Bachmeier, S., Lüring, C., Tingart, M., Püschel, K., Stark, O., Grifka, J., Gehrke, T., Amling, M., Gebauer, M., 2011. Fracture prevention by femoroplastycement augmentation of the proximal femur. J. Orthop. Res. 29, 1753-1758.

https://doi.org/10.1002/jor.21410

Benca, E., Reisinger, A., Patsch, J.M., Hirtler, L., Synek, A., Stenicka, S., Windhager, R., Mayr, W., Pahr, D.H., 2017. Effect of simulated metastatic lesions on the biomechanical behavior of the proximal femur. J. Orthop. Res. 35, 2407-2414. https://doi.org/10.1002/jor.23550

Cazzato, R.L., Palussière, J., Buy, X., Denaro, V., Santini, D., Tonini, G., Grasso, F.R., Zobel, B.B., Poretti, D., Pedicini, V., Balzarini, L., Lanza, E., 2015. Percutaneous Long Bone Cementoplasty for Palliation of Malignant Lesions of the Limbs : A Systematic Review. Cardiovasc. Intervent. Radiol. 38, 1563-1572. https://doi.org/10.1007/s00270-015-1082-7

Coleman, R.E., Roodman, Smith, Body, Suva, Vessella, 2006. Clinical features of metastatic bone disease and risk of skeletal morbidity. Clin. Cancer Res. 12, 6243-6250. https://doi.org/10.1158/1078-0432.CCR-06-0931

Derikx, L.C., van Aken, J.B., Janssen, D., Snyers, A., van der Linden, Y.M., Verdonschot, N., Tanck, E., 2012. The assessment of the risk of fracture in femora with metastatic lesions: Comparing casespecific finite element analyses with predictions by clinical experts. Bone Joint J. 94-B, 1135- 
1142. https://doi.org/10.1302/0301-620X.94B8.28449

Derikx, L.C., Verdonschot, N., Tanck, E., 2015. Towards clinical application of biomechanical tools for the prediction of fracture risk in metastatic bone disease. J. Biomech. 48, 761-766. https://doi.org/10.1016/j.jbiomech.2014.12.017

Deschamps, F., Farouil, G., Hakime, A., Barah, A., Guiu, B., Teriitehau, C., Auperin, A., Debaere, T., 2012. Cementoplasty of metastases of the proximal femur: Is it a safe palliative option? J. Vasc. Interv. Radiol. 23, 1311-1316. https://doi.org/10.1016/j.jvir.2012.06.027

Eggermont, F., Derikx, L.C., Verdonschot, N., van der Geest, I.C.M., de Jong, M.A.A., Snyers, A., van der Linden, Y.M., Tanck, E., 2018. Can patient-specific finite element models better predict fractures in metastatic bone disease than experienced clinicians? Bone Joint Res. 7, 430-439. https://doi.org/10.1302/2046-3758.76.bjr-2017-0325.r2

Eggermont, F., Wal, G. Van Der, Westhoff, P., Jong, M. De, Rozema, T., Kroon, H.M., Derikx, L., Dijkstra, S., Verdonschot, N., Van, Y., 2019. Patient-specific finite element computer models improve fracture risk assessments in cancer patients with femoral bone metastases compared to clinical guidelines. Bone 115101. https://doi.org/10.1016/j.bone.2019.115101

Feng, H., Wang, J., Guo, P., Xu, J., Chen, W., Zhang, Y., 2016. CT-Guided Percutaneous Femoroplasty (PFP) for the Treatment of Proximal Femoral Metastases. Pain Physician 19, E767-73.

Fliri, L., Sermon, A., Wähnert, D., Schmoelz, W., Blauth, M., Windolf, M., 2013. Limited V-shaped cement augmentation of the proximal femur to prevent secondary hip fractures. J. Biomater. Appl. 28, 136-143. https://doi.org/10.1177/0885328212443274

Gilchrist, S., Guy, P., Cripton, P.A., 2013. Development of an Inertia-Driven Model of Sideways Fall for Detailed Study of Femur Fracture Mechanics. J. Biomech. Eng. 135, 121001. https://doi.org/10.1115/1.4025390

Grassi, L., Väänänen, S.P., Amin Yavari, S., Jurvelin, J.S., Weinans, H., Ristinmaa, M., Zadpoor, A.A., 
Isaksson, H., 2014. Full-Field Strain Measurement During Mechanical Testing of the Human Femur at Physiologically Relevant Strain Rates. J. Biomech. Eng. 136, 111010.

https://doi.org/10.1115/1.4028415

Grassi, L., Väänänen, S.P., Ristinmaa, M., Jurvelin, J.S., Isaksson, H., 2016. How accurately can subject-specific finite element models predict strains and strength of human femora? Investigation using full-field measurements. J. Biomech. 49, 802-806. https://doi.org/10.1016/j.jbiomech.2016.02.032

Heini, P.F., Franz, T., Fankhauser, C., Gasser, B., Ganz, R., 2004. Femoroplasty-augmentation of mechanical properties in the osteoporotic proximal femur: A biomechanical investigation of PMMA reinforcement in cadaver bones. Clin. Biomech. 19, 506-512. https://doi.org/10.1016/j.clinbiomech.2004.01.014

Kaneko, T.S., Skinner, H.B., Keyak, J.H., 2007. Feasibility of a percutaneous technique for repairing proximal femora with simulated metastatic lesions. Med. Eng. Phys. 29, 594-601. https://doi.org/10.1016/j.medengphy.2006.06.008

Kok, J., Širka, A., Grassi, L., Raina, D.B., Tarasevičius, S., Tägil, M., Lidgren, L., Isaksson, H., 2019. Fracture strength of the proximal femur injected with a Calcium Sulfate/Hydroxyapatite bone substitute. Clin. Biomech. 63, 172-178. https://doi.org/10.1016/j.clinbiomech.2019.03.008

Kukla, C., Gaebler, C., Pichl, R.W., Prokesch, R., Heinze, G., Heinz, T., 2002. Predictive geometric factors in a standardized model of femoral neck fracture: Experimental study of cadaveric human femurs. Injury 33, 427-433. https://doi.org/10.1016/S0020-1383(02)00076-1

Mirels, H., 1989. Metastatic disease in long bones. A proposed scoring system for diagnosing impending pathologic fractures. Clin Orthop Relat Res 249, 256-264.

Orwoll, E.S., Marshall, L.M., Nielson, C.M., Cummings, S.R., Lapidus, J., Cauley, J.A., Ensrud, K., Lane, N., Hoffmann, P.R., Kopperdahl, D.L., Keaveny, T.M., 2009. Finite element analysis of the 
proximal femur and hip fracture risk in older men. J. Bone Miner. Res. 24, 475-483.

https://doi.org/10.1359/jbmr.081201

Palumbo, B.T., Nalley, C., Gaskins, R.B., Gutierrez, S., Alexander, G.E., Anijar, L., Nayak, A., Cheong, D., Santoni, B.G., 2014. Biomechanical analysis of impending femoral neck fractures: The role of percutaneous cement augmentation for osteolytic lesions. Clin. Biomech. 29, 289-295. https://doi.org/10.1016/j.clinbiomech.2013.12.001

Plancarte-Sanchez, R., Guajardo-Rosas, J., Cerezo-Camacho, O., Chejne-Gomez, F., Gomez-Garcia, F., Meneses-Garcia, A., Armas-Plancarte, C., Saldaña-Ramirez, G., Medina-Santillan, R., 2013. Femoroplasty: A New Option for Femur Metastasis. Pain Pract. 13, 409-415. https://doi.org/10.1111/j.1533-2500.2012.00590.x

Rajapakse, C.S., Gupta, N., Evans, M., Alizai, H., Shukurova, M., Hong, A.L., Cruickshank, N.J., Tejwani, N., Egol, K., Honig, S., Chang, G., 2019. Influence of bone lesion location on femoral bone strength assessed by MRI-based finite-element modeling. Bone 122, 209-217. https://doi.org/10.1016/j.bone.2019.03.005

Ratasvuori, M., Wedin, R., Keller, J., Nottrott, M., Zaikova, O., Bergh, P., Kalen, A., Nilsson, J., Jonsson, H., Laitinen, M., 2013. Insight opinion to surgically treated metastatic bone disease: Scandinavian Sarcoma Group Skeletal Metastasis Registry report of 1195 operated skeletal metastasis. Surg. Oncol. 22, 132-138. https://doi.org/10.1016/j.suronc.2013.02.008

Sternheim, A., Giladi, O., Gortzak, Y., Drexler, M., Salai, M., Trabelsi, N., Milgrom, C., Yosibash, Z., 2018. Pathological fracture risk assessment in patients with femoral metastases using CT-based finite element methods. A retrospective clinical study. Bone 110, 215-220. https://doi.org/10.1016/j.bone.2018.02.011

Sutter, E.G., Mears, S.C., Belkoff, S.M., 2010. A Biomechanical Evaluation of Femoroplasty under Simulated Fall Conditions. J Orthop Trauma 24, 95-9. 
https://doi.org/10.1016/j.jacc.2007.01.076.White

Tian, Q.H., He, C.J., Wu, C.G., Li, Y.D., Gu, Y.F., Wang, T., Xiao, Q.P., Li, M.H., 2016. Comparison of Percutaneous Cementoplasty with and Without Interventional Internal Fixation for Impending Malignant Pathological Fracture of the Proximal Femur. Cardiovasc. Intervent. Radiol. 39, 8189. https://doi.org/10.1007/s00270-015-1133-0

Van der Linden, Y.M., Dijkstra, P.D.S., Kroon, H.M., Lok, J.J., Noordijk, E.M., Leer, J.W.H., Marijnen, C.A.M., 2018. Comparative analysis of risk factors for pathological fracture with femoral metastases. J. Bone Joint Surg. Br. 86-B, 566-573. https://doi.org/10.1302/0301$620 x .86 b 4.14703$

Varga, P., Hofmann-Fliri, L., Blauth, M., Windolf, M., 2016. Prophylactic augmentation of the osteoporotic proximal femur-mission impossible? Bonekey Rep. 5, 1-10. https://doi.org/10.1038/bonekey.2016.86 